\title{
Losoxantrone Hydrochloride
}

National Cancer Institute

\section{Source}

National Cancer Institute. Losoxantrone Hydrochloride. NCI Thesaurus. Code C72077.

The hydrochloride salt form of losoxantrone, an anthrapyrazole-based antineoplastic antibiotic. Losoxantrone intercalates into DNA, induces single- and double-stranded DNA breaks and inhibits topoisomerase II, thereby inhibiting DNA replication and repair as well as RNA and protein synthesis. Losoxantrone is less cardiotoxic than doxorubicin. 